\title{
USING FUZZY LOGIC IN HYBRID MULTIHOP WIRELESS NETWORKS
}

\author{
A.J. Yuste ${ }^{1}$, Alicia Triviño, F.D. Trujillo and E. Casilari ${ }^{2}$ \\ ${ }^{1}$ Department of Telecommunications Engineering, Jaen University, Linares(Jaén), Spain \\ ajyuste@ujaen.es \\ ${ }^{2}$ Department of Electronic Technology, Málaga University, Spain \\ atc, fdtrujillo, ecasilarieuma.es
}

\begin{abstract}
In order to extend the use of Mobile ad hoc networks (MANET) to commercial applications, it is necessary to provide a mechanism to integrate this kind of networks into the Internet. In this way, MANETs could be utilized in visiting theme parks, commercial centers and military scenarios. The interconnection of MANETs and the Internet is supported by a Gateway. The gateway is responsible for informing about some configuration parameters as well as for enabling the creation of the routes to the Internet in the MANET nodes. For these tasks, several control messages are generated. The method in which these messages are originated defines the existing Integration Supports for MANETs. In particular, under the hybrid Global Connectivity support, the Gateway generates periodic Modified Router Advertisements (MRA) which are broadcast in an area close to the Gateway. The optimum values to define the periodicity of these messages and the diameter (number of hops) of the area in which they are propagated depend on the network conditions. Therefore, an automatic and dynamic algorithm is recommended to be implemented in the Gateway to adjust these two parameters. In this sense, this paper presents a technique by which the interval of emission of the MRA messages is controlled by a fuzzy system. The fuzzy system captures several network conditions such as the link stability or the number of sources. The simulation results show that the proposed scheme outperforms other adaptive approaches for the gateway discovery in MANETs.
\end{abstract}

\section{KEYWORDS}

MANET, Internet connection, gateway discovery, adaptive algorithms. Fuzzy logic.

\section{INTRODUCTION}

A Mobile Ad hoc NETwork (MANET) is composed of independent mobile devices that communicate through wireless links. Due to their unrestricted mobility, mobile nodes should constantly self-configure to maintain the routes between nodes. Furthermore, the paths between two nodes could be composed of intermediate nodes that cooperatively retransmit and route the packets to the final destination. Therefore, every node in a MANET performs as a router which can move in any direction and with any speed independently of other nodes' movements. This behavior results in a continuous change of the wireless links that must be taken into account by the protocols executed by the nodes in the MANET.

Due to their ability to work in an autonomous way, MANETs were originally developed for military communications and also for rescue operations where traditional infrastructures are usually not operative. However, the success of wireless communications has prompted the extension of this technology in other scenarios such as conference venues, visiting theme parks or recreation areas. In these situations, different types of user terminals (PDA, laptop, cellular phones) can co-exist. Additionally, these civil applications may be demanded to provide access to external networks, especially to the Internet. The connection between the two networks (the 
MANET and the Internet) is achieved via a special router that acts as the Internet Gateway. When this element is present, the MANET is referred as hybrid mobile ad hoc network. A mobile node in the MANET aiming at connecting to the Internet needs to execute the following tasks:

- Identification and Selection of the Internet Gateway. To identify the available Gateways, the Gateways emit specific messages. The messages can be generated periodically or on demand. On the other hand, the selection of the Internet Gateway is usually based on the number of hops that the routes to the Gateway contain. However, other parameters could be taken into account such as the traffic load or the delay in the route.

- Construction of a reachable IP address. The method for generating an IP address could be stateless (nodes are responsible for their own IP address) or stateful (supported by an entity which distributes the IP addresses in the network).

- A duplicated Address Detection (DAD) operation is also necessary when a stateless mechanism is employed to construct the IP address. This permits to guarantee that the chosen IP address is unique in the network.

This paper focuses on the first phase of the interconnection, that is, the identification of the available Gateways in the network. For this purpose, we take into account that the Gateway has two main tasks. Firstly, it is capable to route the packets from the Internet to a MANET node. Conventional Access Routers do not implement any ad hoc routing protocol so they need to be assisted by an Internet Gateway. On the other hand, the Gateway informs about the configuration parameters by generating Modified Router Advertisement (MRA) messages. Upon reception, the nodes also create, update or optimize the route to the Internet Gateways which are employed to send the packets to the Internet hosts. The process by which these messages are generated characterizes the integration supports [1] [2] [3]. Among them, the Global Connectivity support [2] is the most popular one. In this scheme, discovery processes may be classified intro three main categories: proactive, reactive and hybrid. Under the reactive discoveries, the nodes only search for a route to the Internet Gateway (IGW) when they have some data to send to external hosts so they do not constantly keep a valid route to the IGW. To find the route to the Gateway, the node generates a Modified Router Solicitation (MRS) message which is replied by the Internet Gateway with a unicast MRA message. Conversely, the proactive algorithms are characterized by the periodic emission of MRA messages by the IGW. These MRA messages are periodically broadcast to all the nodes in the MANET every $T$ seconds. After receiving a MRA message, the changes on the wireless links could make the route to the Gateway invalid. Under these circumstances, the nodes will operate as in the reactive scheme. Therefore, when needed, it will emit a MRS message and it will wait for a unicast MRA message. Finally, the hybrid schemes combines the two previous approaches as the MRA messages are periodically generated in an area close to the Gateway. Conversely, the nodes outside this zone update the route to the IGW in a reactive way. In the hybrid approach, the proactive zone is defined by the TTL (Time To Live) value of the IP (Internet Protocol) header in the MRA message [4]. This parameter restricts the number of forwardings that the message can have. By adjusting the TTL value, the hybrid gateway discovery can behave as proactive (the TTL is set to the network diameter) or as reactive (if the TTL is set to 0).

The three types of gateway discovery processes generate control messages (MRA or MRS). In order to improve the network performance, it is important to reduce the number of these control messages while keeping valid routes to the Gateways in the nodes that need them. By this way, the scarce wireless resources are released to the data packets which experiment lower delay and losses. Since hybrid gateway discovery is able to emulate all gateway discovery schemes, in this 
paper we will focus on optimizing it. However, tuning the values of the TTL and the $T$ parameters is not a trivial task in conventional MANET applications. Specifically, the optimum value for the $T$ parameter depends on several factors such as the number of traffic sources, their positions, their route duration or the density of nodes [5]. Furthermore, as network conditions are expected to vary unpredictably in actual MANET scenarios, an autonomous and dynamic algorithm to adjust the $T$ and TTL parameters is strongly recommended.

The main drawback to overcome is the fact that there is not any mathematical association that relates these factors with the optimum value of $\mathrm{T}$ setting. Thus, our proposed scheme dynamically adapts the value of $T$ by a fuzzy algorithm. MANETs can benefit from fuzzy systems as they are able to capture generic behaviors of the MANET according to the parameters settings. For instance, we can state that a stable MANET needs less MRA messages than a MANET with continuous link changes. Constructing several similar rules, the fuzzy system has shown to improved the network performance in terms of packet losses, delay and control overhead.

The rest of the paper is structured as follows. In Section 2, we present a detailed description about other adaptive techniques. Section 3 explains our algorithm which is evaluated by simulations presented in Section 4. Finally, Section 5 draws the main conclusions of our work.

\section{RELATED WORK}

The hybrid gateway discovery possesses two parameters to be configured: the interval of emission of the MRA messages $(T)$ and the radius of the area in which these messages are periodically broadcast (TTL).

Concerning the adjustment of the TTL parameter, one of the first proposals was the Maximal Source Coverage (MSC) [6]. In this proposal, the Gateway sends the advertisement message with the TTL set to the minimum number of hops required to reach all the sources that are employing this gateway to communicate with the Internet. An optimized scheme of the MSC algorithm is presented in [7]. In this approach, the nodes that are delimiting the proactive zone act as proxy for the Internet Gateway so they can reply to the MRS messages with unicast MRA messages. The scheme is known as Low Overhead and Scalable Proxied (LOSP) algorithm. Alternatively, the work in [8] presents a novel technique in which the decision about forwarding the MRA messages is transferred to the MANET nodes. In particular, just the mobile nodes that are relaying data packets are allowed to forward the MRA messages. In this sense, the area of the proactive zone is decided in a distributed way.

On the other hand, the work in [9] analyzes the adjustment of the $T$ parameter. It presents an algorithm in which the appropriateness of broadcasting an MRA message depends on the number of active sources that communicate with the Internet as well as the number of intermediate nodes that are employed for these communications. With these two parameters, the authors defined the so-called Regulated Mobility Degree (RMD). If this factor exceeds a threshold, the algorithm considers that a MRA is needed and, in turn, the message is sent. The main drawback of this solution is deciding the threshold as its optimum value also depends on the network conditions. This issue is not studied in the paper and the parameterization of the threshold is heuristic.

Focusing on the simultaneous tuning of the $T$ and TTL parameters, the authors in [10] propose the use of an auto-regressive filter. Then, the traffic load in the Internet Gateways is monitored to identify the traffic rate and the number of received MRS messages. Taking into account these two parameters and the number of link changes, the system is expected to improve the network 
performance. The main drawback of this proposal is that there is not any specific formulation and no evaluation is shown.

As we can see, the proposed algorithms are supported by different metrics. The complexity of using these metrics relies on the absence of a mathematical model that defines the relationship between the analysed metric and the resulting overhead. Fuzzy logic provides a mechanism to cope with this type of uncertainty. This is why fuzzy logic is widely employed in telecommunication applications including MANETs. In this sense, [11] uses an adaptive fuzzy logic algorithm that adapts the security functionalities to the dynamic conditions of mobile nodes. Similarly, [12] proposes a fuzzy based algorithm in the routing procedures for MANETs. In particular, the fuzzy logic is employed to select the appropriate path in a multiple path routing protocol based on a QoS (Quality-of-Service) metric. Furthermore, [13] has demonstrated that fuzzy logic can be implemented in devices with reduced resources such as sensors. In this paper, we use a fuzzy logic system intended to optimize the timing of the MRA messages. The use of a controller to adapt the interval of emission of the MRA messages is not novel. In this sense, we have already used a controller that was configured by means of the statistical properties of the movement in the MANET [14]. In particular, this configuration was exclusively valid for a Random WayPoint mobility model so it is difficult to extend this study to other mobility patterns. A fuzzy logic-based controller was presented in [15]. The proposed system was supported by measurements that the Gateway can make when it emits the MRA messages. Therefore, only the information about the system when the MRA messages are emitted could be taken into account. Reducing the analysis to these events seems to be inadequate in highly dynamic scenarios.

\section{Proposed Gateway Discovery}

Our proposed scheme is supported by a fuzzy system which can use several parameters informing about the MANET conditions. In particular, the system takes into account the following parameters:

- The number of received MRS (NMRS). It is defined as:

$$
N M R S=\frac{\text { number of MRS messages }}{\text { number of traffic sources }}
$$

Therefore, it represents the ratio between the number of MRS messages that the sources originate and the number of active sources. To make the Gateway able to compute this parameter, the MRS messages are only replied by the Internet Gateway. This metric was used in [16].

- The link changes (LC). The mobility near the Internet Gateway is measured as the number of link changes that the Gateway detects divided by the number of traffic sources that are using this gateway. It is computed as:

$$
L C=\frac{\text { number of link changes }}{\text { number of traffic sources }}
$$

With this parameter, the mobility around the Internet Gateway is considered. This metric has been employed in different studies. For instance, [17] proposes the rate of link changes 
as a metric to differentiate the mobility conditions when nodes follow the RPGM (Reference Point Group Mobility) and RWP (Random WayPoint) models.

- The TTL changes (TTLC). It represents the number of changes in the distance of the sources to the Gateways. The distance is measured as the number of nodes (hops) that the messages have to traverse to reach the final source from the Internet Gateway. This distance can be inferred from the TTL field in the data packets that the sources generate to the Internet. As all the traffic flowing from/to the Internet is routed by the Internet Gateway, this element can easily become aware of the distance to the nodes with Internet connections. In particular, the average value of the TTL changes is computed as expressed in the following equation:

$$
T T L C=\frac{\text { number of TTL changes }}{\text { number of traffic sources }}
$$

This metric provides a hint about the mobility of the MANET nodes which are not directly connected to the Gateway. To the best of the authors' knowledge, this metric has not been employed previously.

These three parameters are computed (and updated) every second. In order to introduce them into the fuzzy system, they need to be fuzzified. Particularly, the input variables have three possible variables: low, moderate and high. The selected membership functions are represented in Figure 1. We have used a triangle-based function since this type of functions has been extensively used in the real time applications due to their simple formulas and their computational efficiency.

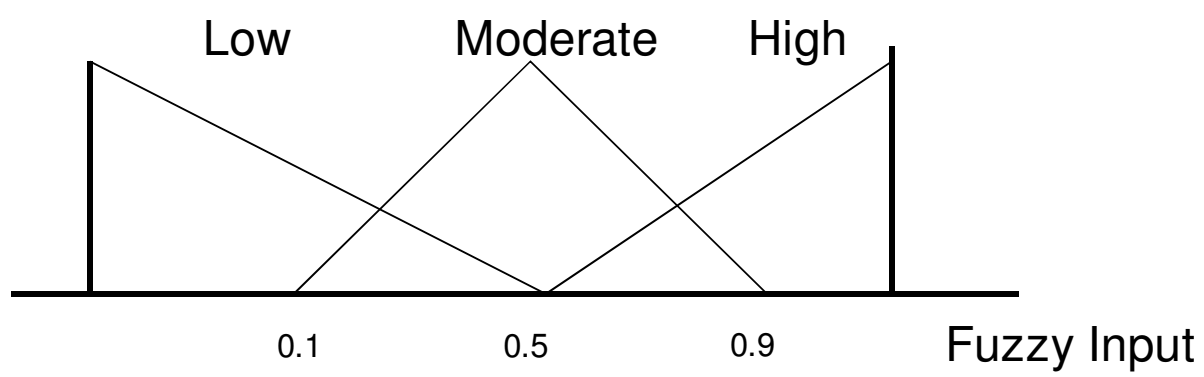

Figure 1. Membership function of the inputs for the fuzzy system.

As we can observe, the inputs are assumed to have a value from 0 to 1 . It could happen that one of the used parameter is greater than one. In this case, the affected parameter is set to 1 so that the system can cope with it.

From the inputs, the application applies some fuzzy rules into an inference system. The fuzzy rules for our system are presented in Table 1. 
Table 1. Fuzzy rules for the proposed system to adapt the $T$ parameter.

\begin{tabular}{|l|l|l|l|}
\hline $\begin{array}{l}\text { Number of MRS } \\
\text { (NMRS) }\end{array}$ & $\begin{array}{l}\text { Link Changes } \\
\text { (LC) }\end{array}$ & $\begin{array}{l}\text { TTL Changes } \\
\text { (TTLC) }\end{array}$ & Convenience \\
\hline Low & Low & Low & Very Low \\
\hline Low & Low & Moderate & Very Low \\
\hline Low & Low & High & Low \\
\hline Low & Moderate & Low & Very Low \\
\hline Low & Moderate & Moderate & Low \\
\hline Low & Moderate & High & Moderate \\
\hline Low & High & Low & Low \\
\hline Low & High & Moderate & Moderate \\
\hline Low & High & High & High \\
\hline Moderate & Low & Low & Very Low \\
\hline Moderate & Low & Moderate & Low \\
\hline Moderate & Low & High & Moderate \\
\hline Moderate & Moderate & Low & Low \\
\hline Moderate & Moderate & Moderate & Moderate \\
\hline Moderate & Moderate & High & High \\
\hline Moderate & High & Low & Moderate \\
\hline Moderate & High & Moderate & High \\
\hline Moderate & High & High & Very High \\
\hline High & Low & Low & Low \\
\hline High & Low & Moderate & Moderate \\
\hline High & Low & High & High \\
\hline High & Moderate & Low & Moderate \\
\hline High & Moderate & Moderate & High \\
\hline High & Moderate & High & Very High \\
\hline High & High & Low & High \\
\hline High & High & Moderate & Very High \\
\hline High & High & Very High \\
\hline
\end{tabular}

Then, the fuzzy system obtains an output called convenience as it represents the appropriateness of emitting a MRA message. The membership of the variable convenience is represented in the Figure 2.

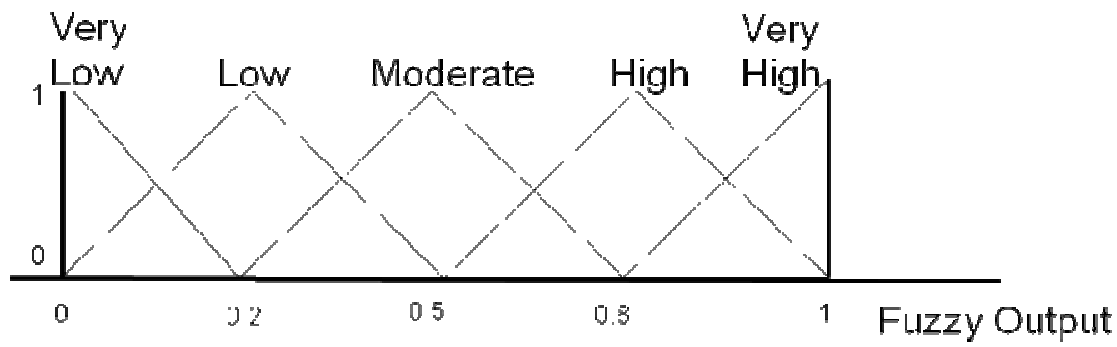

Figure 2. Membership functions of the output (called convenience) for the fuzzy system.

Once the output is computed, the Gateway compares it to a predetermined threshold (set to 0.5 in our experiments). When it exceeds the threshold, the Gateway considers that a MRA message is necessary in the network and, consequently, it broadcasts a new one. Under these 
circumstances, the process to decide about the emission of new MRA messages is paused along 3 seconds. By this interruption, the MRA messages, which are an extended version of Router Advertisement (RA) messages, also follows the recommendations presented in [18]. On the contrary, the decision phase is triggered every second with updated measurements. The parameters are measured each second so that the algorithm is able to adapt itself to rapid changes in the movements of nodes. By this, routes to the Gateway remain stable longer so the overhead is reduced. Figure 3 summarizes the scheme of the fuzzy system.

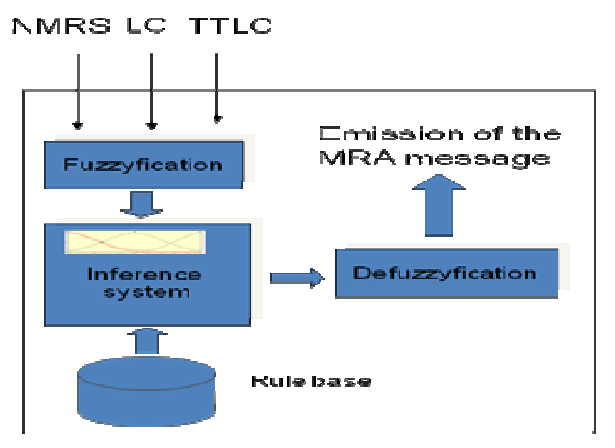

Figure 3. Decision on the emission of the MRA message.

The presented fuzzy system controls the interval of emission of the MRA messages. As we intend to apply it in a hybrid gateway discovery scheme, the TTL value is adjusted according to the MSC strategy due to its simplicity and its good results. Although the LOSP is an improved version of the MSC, it is not appropriate for the proposed scheme as it does not allow the Gateway to compute the parameter "Number of MRS".

\section{EVALUATION RESULTS}

The evaluation of our proposal has been conducted by a comparative approach. In order to analyze different scenarios but keeping the same conditions for all the evaluated algorithms, the use of a simulation tool becomes necessary. In this sense, our study is supported by the Network Simulator tool ns-2.31 [19]. The network Simulator tool is widely used in MANET research. For instance, it is in the evaluation of security protocols [20], of multicast data transmissions [21] and routing [22]. As a previous step, the tool was extended with the Global connectivity support [23] and the evaluated schemes. In particular, we have compared our scheme, called the Fuzzy-based Gateway Discovery (FGD), to LOSP, ADD and RMD (all these algorithms are described in the Related Work). By comparing to LOSP, we can evaluate the goodness of the proposed fuzzy system to tune the interval of emission of the MRA messages (the T parameter). It is important to note that FGD also adapts the TTL value by means of the MSC scheme, which is a simplified version of the LOSP scheme. On the other hand, ADD represents a distributed algorithm which controls the forwarding of the MRA messages by the MANET nodes. Finally, RMD focuses on controlling the $T$ parameter taking into account some network conditions. Comparing the results of FGD to RMD and ADD, we can decide about the appropriateness of adjusting the $T$ and TTL parameters simultaneously. The RMD is configured according to [9].

The performance of the MANET is evaluated with the following metrics:

- Packet Loss Rate. It is defined as the ratio between the number of lost data packets and the total number of data packets transmitted by the sources. 
- Normalized Routing Overhead. It represents the number of control packets divided by the total number of received data packets. For this computation, every time a control packet is retransmitted, it is considered as a new control packet.

The simulation area is a rectangle of $1500 \times 300 \mathrm{~m} 2$ where nodes move according to the Timevariant Community Mobility Model (TVCM) mobility pattern [24]. This is a realistic model obtained from traces of wireless LAN (Local Area Networks). It includes two mobility characteristics: skewed location visiting preferences and periodical re-appearance. It also incorporates communities that the mobile nodes often visit. In our scenarios, we define two random communities. The maximum speed of the nodes is set to $20 \mathrm{~m} / \mathrm{s}$.

Concerning the traffic, we have varied the number of Constant Bit Rate (CBR) sources in our simulations. However, they all emit at a traffic rate of 10 packet/s with a packet length of 320 bytes.

In all the scenarios, the simulation time is set to 1000 seconds. Since we are interested in studying the behavior of MANETs in a steady state, the first 100 seconds of the simulations are considered a warm-up period and, consequently, they are not taken into account for the analysis. For each analyzed condition and algorithm, the simulations are repeated 30 times. The figures show the averaged results.

For the evaluation, we have varied two basic parameters: the number of nodes and the number of sources. By this way, we pretend to evaluate the goodness of our proposal in a wide spectrum of scenarios. Table 2 summarizes the parameters of the simulations.

Table 2. Simulation Parameters.

\begin{tabular}{|c|c|}
\hline Simulation Area & $1500 \times 300 \mathrm{~m}^{2}$ \\
\hline $\begin{array}{c}\text { Transmission } \\
\text { Range }\end{array}$ & $250 \mathrm{~m}$ \\
\hline Simulation Time & $1000 \mathrm{~s}$ \\
\hline Runs per point & 30 \\
\hline $\begin{array}{c}\text { Integration } \\
\text { Support }\end{array}$ & $\begin{array}{c}\text { Global Connectivity } \\
\text { Local Repair disabled } \\
\text { Link Layer feedback activated }\end{array}$ \\
\hline Link Layer & $802.11 \mathrm{~b}$ \\
RTS/CTS enables
\end{tabular}

Firstly, we vary the number of nodes in the MANET from 40 to 55. For this case, we assume that there are 40 traffic sources and 4 Gateways. The Gateways share the same prefix so a MANET node could use any Gateway. The Gateways are placed in the corners of the simulation area. Figure 4 shows the packet loss rate and the normalized overhead for this kind of scenarios. 

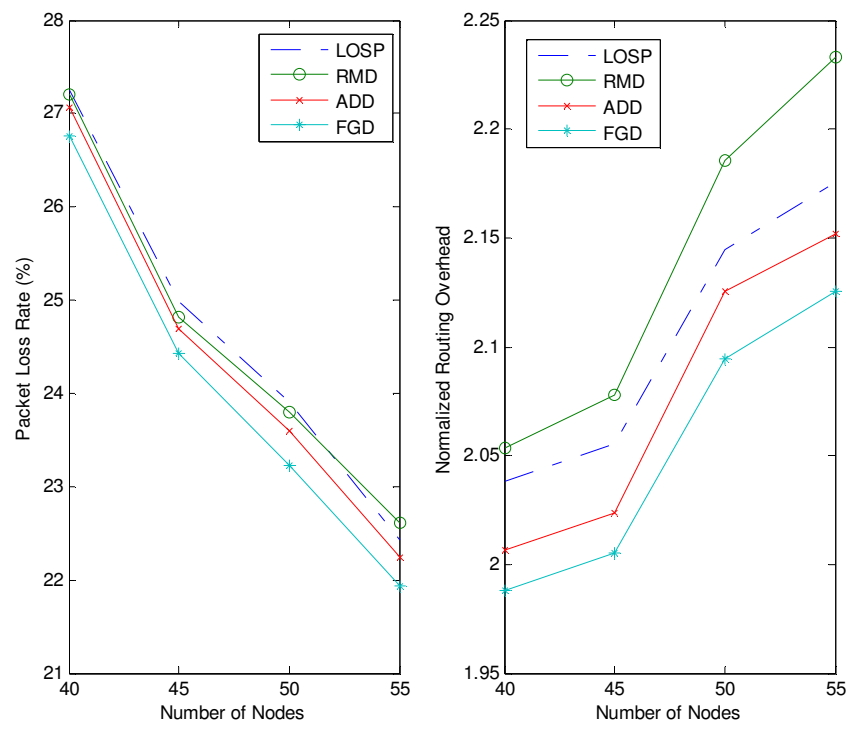

Figure 4. Packet Loss Rate and Normalized Overhead as a function of the number of nodes.
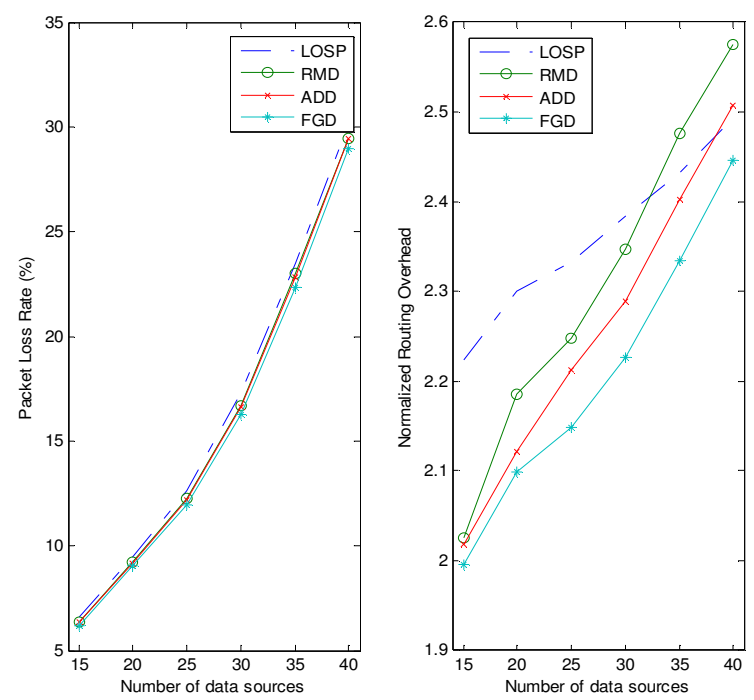

Figure 5. Packet Loss Rate and Normalized Overhead as a function of the number of data sources.

As we can see, incrementing the number of nodes reduces the packet losses. This is due to the improvement of the gateway connectivity which leads to more available routes to the Internet Gateway. However, the routes are composed of a higher number of nodes which provokes that the lifetimes of the paths are lower. In turn, the MANET nodes need to generate more control messages to discover alternative routes to the Gateway. This explains why the normalized 
routing overhead is incremented for a higher number of nodes. In the analyzed conditions, FGD outperforms the other proposals.

Another set of experiments consists in analyzing the impact of the number of traffic sources. To proceed, we placed 2 Gateways at the opposite corners of the simulation area. In this case, the MANET is composed of 50 nodes and the number of sources varies from 15 to 40 . Figure 5 represents the obtained results for the packet loss rate and the normalized overhead.

Figure 5 shows that there is little difference in the packet loss rate obtained by the evaluated techniques. Losses always increase when the number of data sources is incremented. When augmenting the traffic sources, more nodes need updated routes to the Internet. If they do not possess a valid route, they will emit a MRS message to obtain an alternative route. This behavior will induce extra overhead as represented in the Figure. Concerning the overhead, the FGD is able to reduce the number of control messages while keeping an acceptable packet loss rate.

In our last experiment, we change the number of Gateways (from 1 to 6). For this test, Gateways were located in the positions summarized in Table 3. The number of traffic sources is set to 20 and there are 50 mobile nodes. Results are shown in Figure 6.

Table 3. Location of the gateways.

\begin{tabular}{|c|c|}
\hline Location & Coordinates \\
\hline 1 & $(0,0)$ \\
\hline 2 & $(1500,300)$ \\
\hline 3 & $(0,300)$ \\
\hline 4 & $(1500,0)$ \\
\hline 5 & $(750,0)$ \\
\hline 6 & $(750,300)$ \\
\hline
\end{tabular}

As we can observe, the increment in the number of gateways leads to a reduction in terms of losses and overhead. This reduction is similar in the adaptive techniques that have been studied in our work.

Compared to the LOSP and the ADD, the performance of the MANET with the FGD is better. This behavior reinforces the need for adaptive techniques that controls the $\mathrm{T}$ parameter. However, this adjustment must be done with the TTL value as the results improve. This effect can be observed if we compared the results of the FGD and the RMD algorithms. 

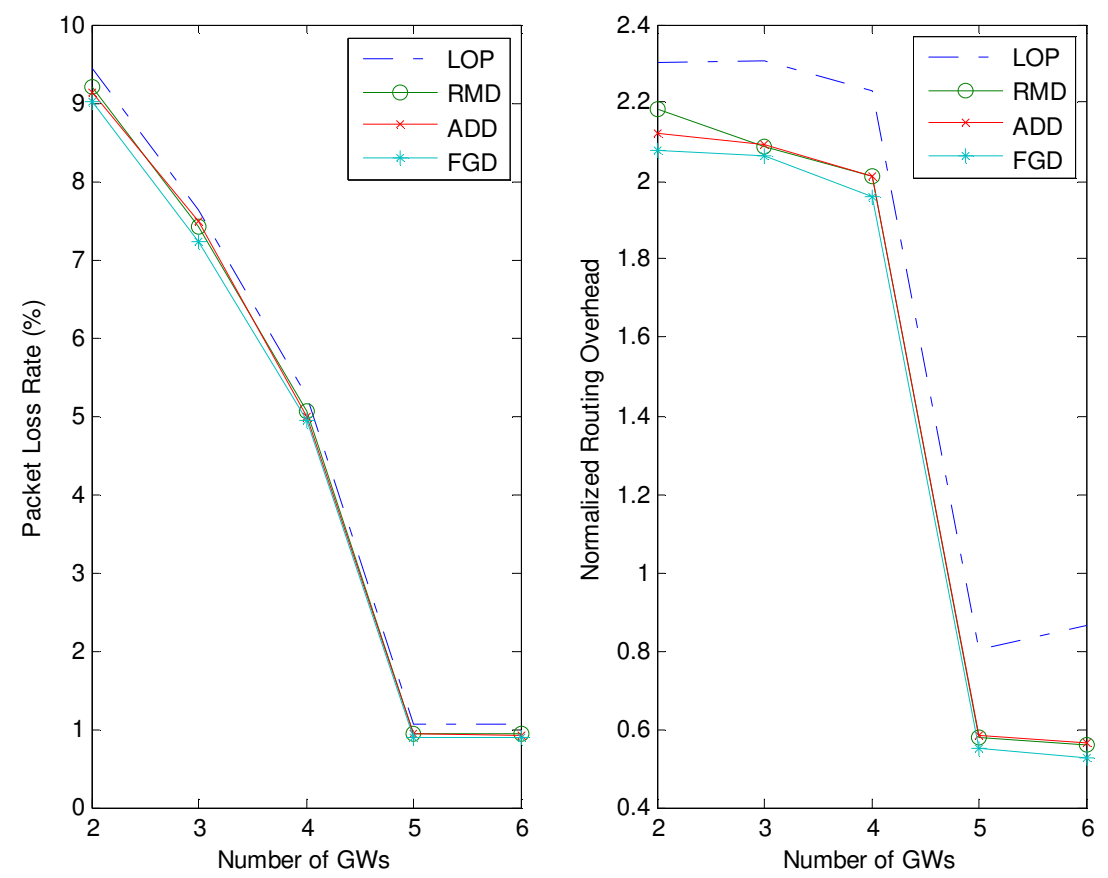

Figure 6. Packet Loss Rate and Normalized Overhead as a function of the number of gateways.

\section{Conclusions}

The integration of a MANET into the Internet is achieved by an Internet Gateway which emits MRA messages. There exist different methods to generate the MRA messages depending on the Integration Supports. In the hybrid Global Connectivity support, the messages are generated periodically in an area close to the Gateway. This paper presents a technique by which the dimension of this area (in terms of the number of hops from the Gateway) and the periodicity of the Gateway advertisements are controlled. In particular, the timing of the MRA messages is controlled by a fuzzy logic-based system. The fuzzy system takes into account the number of link changes, the movement of the traffic sources and the number of solicitations that the sources generate. The simulation results show the goodness of our proposal in terms of packet loss rate and normalized overhead. In our future work, we aim at introducing the fuzzy logic in the mobile nodes so that they can decide about the appropriateness of retransmitting the MRA messages. In this way, the TTL parameter of the MRA messages will be dynamically adjusted.

\section{ACKNOWLEDGEMENTS}

This work has been partially supported by national public funds by the Spanish National Project No. TEC2009-13763-C02-01.

\section{REFERENCES}

[1] Jelger, C., Noel, T. and Frey, A. (2006). "Gateway and address autoconfiguration for IPv6 ad hoc networks (work in progress)", IETF Internet draft (2006).

[2] Wakikawa, R., Malinen, J., Perkins, C., Nilsson, A. and Tuominen, A. (2006). "Global Connectivity for IPv6 Mobile Ad Hoc Networks (work in progress)”, IETF Internet draft . 
[3] Ruffino, S. and Stupar, P. (2006). "Automatic configuration of IPv6 addresses for MANET with multiple gateways (work in progress)", IETF Internet draft .

[4] Defense Advanced Research Project Agency (1981). “Internet Protocol, IET RFC 791”.

[5] Yuste, A.J., Triviño, A., Trujillo, F.D., Casilari. E. and Díaz-Estrella, A. (2008). “ Connectivity Gateway discovery in MANETS”, Lecture Notes in Computer Science 5122, pp. 128-141

[6] Ruiz, P. and Gomez-Skarmeta, A. (2004). "Maximal Source coverage adaptive gateway discovery for hybrid ad hoc networks", Lecture Notes in Computer Science, 3158, pp. 28-41.

[7] Ros, F. and Ruiz, P. (2006). "Low Overhead and Scalable Proxied Adaptive Gateway Discovery for Mobile Ad Hoc Networks", IEEE International Conference on Mobile Adhoc and Sensor Systems (MASS), pp. 226-235.

[8] Javaid, U., Rasheed, F., Meddour, E.E and Ahmed, T. (2008). “Adaptive distributed gateway discovery in hybrid wireless networks", IEEE Wireless Communications and Networking Conference (WCNC), pp. 2735-2740.

[9] Rakeshkumar, V. and Misra, M. (2006). “An Efficient Mechanism for Connecting MANET and the Internet through Complete Adaptive Gateway Discovery", First International Conference on Communication System Software and Middleware, pp. 1-5.

[10] Ghassemian, M., Friderikos, V. and Aghvami, A. (2006). " A Generic Algorithm to Improve the performance of proactive ad hoc mechanisms", IEEE International Symposium on a World of Wireless, Mobile and multimedia Networks.

[11] Nie, J. , Wen, J., Luo, J.,He, X. and Zhou, Z. (2006). "An adaptive fuzzy logic based secure routing protocol in mobile ad hoc networks", Fuzzy sets and systems, vol. 157, n 12, pp 17041712.

[12] Zhang, X., Sheng Cheng, Mei-yu Feng and Wei Ding. (2004). "Fuzzy Logic QoS Dynamic Source Routing for Mobile Ad Hoc Networks," International Conference on Computer and Information Technology, pp. 652-657.

[13] Canada-Bago, J., Fernandez-Prieto, J.A., Gadeo-Martos, M. A. and Velasco, J.R. (2010). “A New Collaborative Knowledge-Based Approach for Wireless Sensor Networks", Sensors 10, no. 6. pp. 6044-6062.

[14] Yuste, A.J., Triviño, A., Trujillo, F.D. and Casilari, E. (2010). "Improved Scheme for Adaptive Gateway Discovery in Hybrid MANET", International Conference on Distributed Computing Systems Workshops, pp. 270-275.

[15] Yuste, A.J., Triviño, A., Trujillo, F.D., Casilari, E. and Díaz-Estrella. A. (2009). "An adaptive genetic fuzzy control gateway discovery to interconnect hybrid MANETs", IEEE conference on Wireless Communications \& Networking Conference, pp. 2810-2815.

[16] Yuste, A.J., Triviño, A., Trujillo, F.D., Casilari, E. and Díaz-Estrella. A. (2009). “Optimized gateway discovery in hybrid manets", International Journal of Computer Networks \& Communications (IJCNC), Vol.1, No.3, October 2009, pp. 78-91.

[17] Hong, X.,Gerla, M., Pei, G. and Chiang, C.C. (1999). “A group mobility model for ad hoc wireless networks", ACM international workshop on Modeling, analysis and simulation of wireless and mobile systems, pp. 53-60.

[18] Narten, T., Nordmark, E. and Simpson, W. (1998). "Neighbor Discovery for IP Version 6, IETF RFC 2461".

[19] Fall, K. and Varadhan, K. (2009). " ns Notes and Documentation, The VINT Project”.

[20] Iyengar, N. Ch. Sriman Narayana, kumar, Syed Mohammad Ansar Sachin, Nagar, Piyush, Sharma, Siddharth and Atrey, Akshay (2010). "An Efficient and Secure Routing Protocol for Mobile Ad-Hoc Networks". International Journal of Computer Networks \& Communications (IJCNC), May 2010, Volume 2. Number 3, pp 28-36 
[21] Manoharan, R. and Ilavarasan, E. (2010). "Impact of Mobility on the Performance of Multicast Routing Protocols in MANET”. International Journal of Wireless \& Mobile Networks (IJWMN), May 2010, Volume 2, Number 2, pp . $110-119$

[22] Gao, Longxiang and Li, Ming. (2009). "Virtual Backbone Content Routing in Wireless Ad-Hoc Network" International Journal of Wireless \& Mobile Networks (IJWMN), Vol 1, No 2, November 2009, pp. $33-47$.

[23] Hamidian, A., Körner, U. and Nilsson, A (2004). "Performance of Internet Access solutions in mobile ad hoc networks", EuroNGI Workshop, pp. 189-209.

[24] Hsu, W., Spyropoulos, T., Psounis, K. and Helmy, A. (2007). "Modeling time-variant user mobility in wireless mobile networks", IEEE Infocom, pp. 758-766.

\section{Authors}

A. J. Yuste was born in Málaga, Spain, in 1970. He received the MSc in Telecommunication Engineering from University of Málaga in 1994. Since 2003, he is associate professor at Telecommunication Engineering Department of Jaén University. His area of research interests are routing in ad hoc networks, grid computing and traffic analysis of computer networks. He is involved in financed research projects of the Spanish Ministry of Science and Education and private companies.

Alicia Triviño-Cabrera was born in 1978. After finishing her studies on Telecommunication Engineering in 2002, she started to work at the Department of Electronic Technology (University of Málaga, Spain). In 2003, she was granted with a scholarship for coursing her $\mathrm{PhD}$ studies, which she finished in 2007. Along these studies, she had the opportunity to work at Samsung Advanced Institute of Technology in South Korea and at the Laboratory of Communications and Telematics of the University of Coimbra in Portugal. She is currently working at the University of Malaga as an assistant professor. Her research areas include modelling and routing in mobile ad hoc networks and wireless mesh networks.

F. D. Trujillo was born in Málaga, Spain, in 1973. He received the BS degree in Telecommunication Engineering from University of Málaga in 1997. Since then to 2002, he worked as assistant professor in the Electronic Technology Department of the University of Málaga and in December 2002, he became an associate professor in the same Department. Nowadays, he is performing his $\mathrm{PhD}$ studies, being involved in several financed research projects and private companies. His research interests cover several areas like the routing in ad hoc networks; the presence in virtual reality and the European Higher Education Area.

Eduardo Casilari (born in 1972) received the B.S. degree (1995) and the Ph.D. degree (1998) in electrical (Telecommunications) engineering, both from the University of Málaga (Spain). From 1996 to 2001 he worked as an assistant professor in the Department of Electronic Technology (University of Málaga, Spain). In September 2001 he became an associate professor in the same University. His works have been partially published in international congresses and publications (IEE Electronic Letters, IEEE Communications Letters). His current research areas include ad hoc networking and Personal Area Networks.
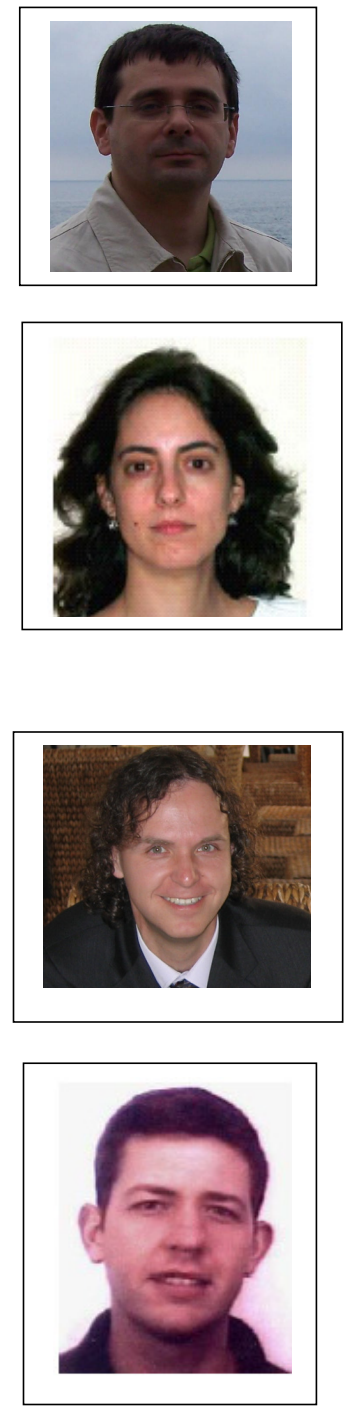